\title{
Gpyro3D: A Three Dimensional Generalized Pyrolysis Model
}

\author{
CHRIS LAUTENBERGER \\ Reax Engineering Inc. \\ 1921 University Ave. \\ Berkeley, CA 94704
}

\begin{abstract}
All objects that burn in fires are three dimensional. However, almost all previous attempts to model the burning or pyrolysis of real materials has resorted to a one-dimensional description. This paper presents the mathematical formulation of Gpyro3D, a generalized three dimensional pyrolysis model that solves conservation equations for transport of heat, mass, and species in a chemically reacting porous medium, as well as its coupling to NIST's Fire Dynamics Simulator (FDS) for simulating fire development. Gpyro3D facilitates pyrolysis modeling in complex geometries by masking grid cells on a regular Cartesian grid, similar to the way that geometry is specified in FDS. The model's mathematical formulation is verified by comparing numerical simulations to analogous exact solutions. Next, it is shown that Gpyro3D qualitatively captures the major three dimensional features of long-duration $(>1$ hour) oxidative pyrolysis of wet wood under radiative heating. Finally, fire development in a wood crib is simulated with the coupled Gpyro3D/FDS model. By observing condensed-phase temperature contours within burning elements of the wood crib, it is shown that strong three dimensional effects are present.
\end{abstract}

KEYWORDS: pyrolysis, flame spread, modeling

\section{NOMENCLATURE LISTING}
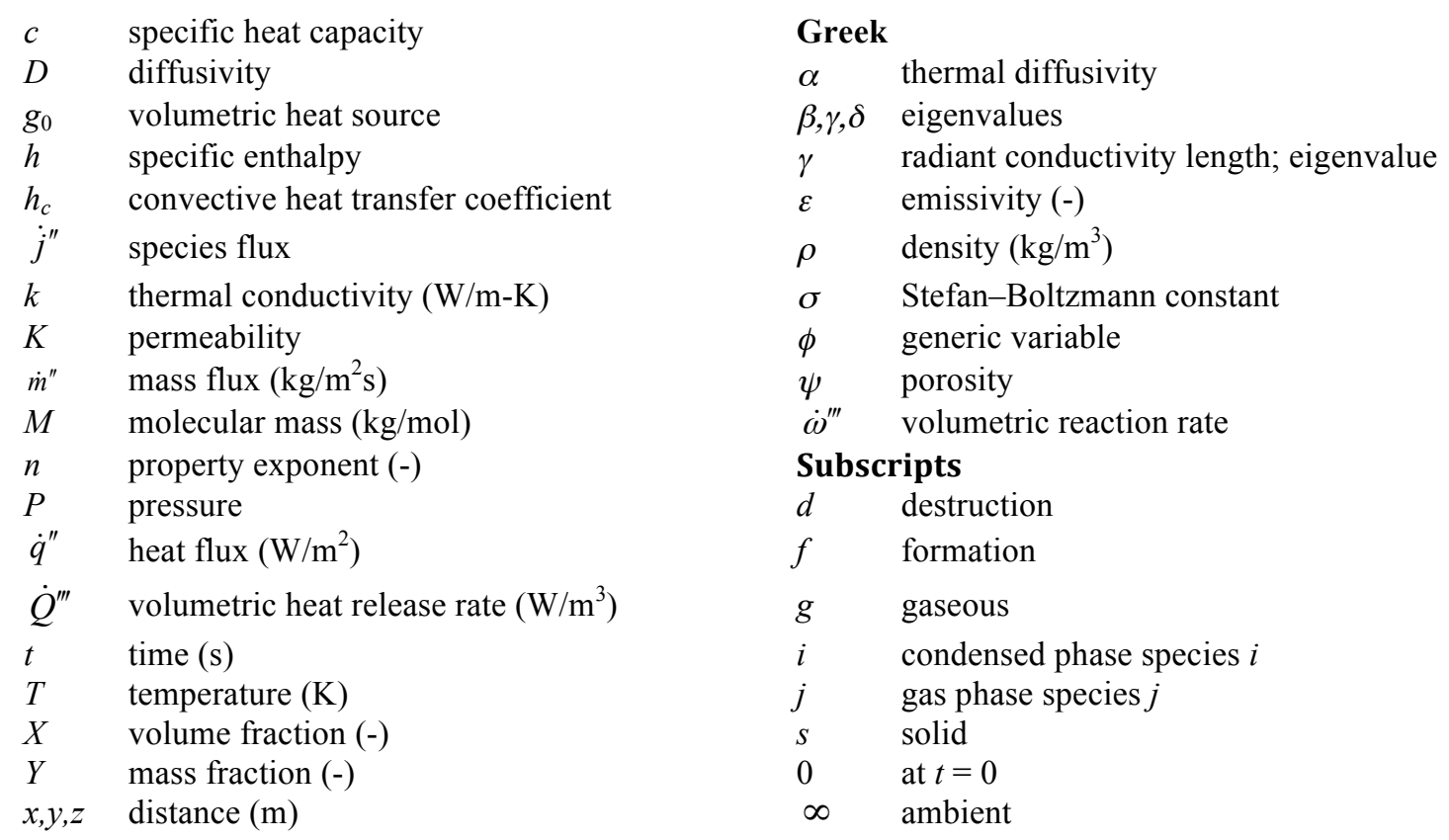

\section{INTRODUCTION}

Current fire models such as Fire Dynamics Simulator [1] and FireFOAM [2] include condensed-phase pyrolysis models that facilitate prediction of HRR. Historically, the biggest hurdle to simulating fire spread with such models has been the difficulty associated with determining the material properties of practical materials. This process, sometimes called "property estimation" or "parameter estimation", involves applying optimization techniques to extract material pyrolysis properties from conventional flammability tests such as the Cone Calorimeter or Fire Propagation Apparatus; specialized bench-scale tests can also be used to estimate material pyrolysis properties. 
While considerable progress has been made in this area in recent years, the material pyrolysis property estimation problem has not yet been completely solved. One difficulty associated with material pyrolysis property estimation is that the level of "physics complexity" must be specified by the user a priori by deciding whether to include or omit certain phenomena. Toward the "simple" end of the physics complexity spectrum, wood charring can be modeled as conversion of an opaque, homogeneous, constantproperty solid to a lower density homogeneous constant property residue by a single-step first order Arrhenius reaction. At the other end of the physics complexity spectrum, wood charring can be modeled as conversion of an anisotropic semi-transparent solid to anisotropic porous residues, all having temperaturedependent thermal properties, with the conversion rate controlled by multiple oxidative and non-oxidative Arrhenius reactions occurring in parallel and serial. The complex approach would require specification of 3-4 times more material properties than the simple approach. The optimal level of physics complexity (which provides reasonably accurate predictions without including non-essential physical processes that increase the number of input parameters) lies somewhere between these two extremes. Bal and Rein recently [3] addressed balancing physics complexity with accuracy for pyrolysis of thermoplastics.

Although the concept of appropriate physics complexity in pyrolysis modeling has been partially addressed, the concept of appropriate geometrical complexity in pyrolysis modeling remains almost completely unexplored. Although hundreds of papers that address various aspects of pyrolysis modeling can be found in the literature, only a few studies have formulated and applied three-dimensional models, e.g. Refs. [4-8]. Most likely because wood is such a ubiquitous part of the built environment and also has importance for energy conversion and recovery, the published 3D pyrolysis model formulations [4-8], with the notable exception of McGurn et al. [8], emphasize wood. It does not appear that a generalized (meaning applicable to thermoplastics and charring solids) 3D pyrolysis model has been formulated and demonstrated. Furthermore, there appear to be few investigations of pyrolysis modeling in complex three dimensional (i.e. non-parallelepiped) geometries; the same can be said about using a 3D pyrolysis model coupled to a gas-phase fire model to simulate fire development.

This paper presents the mathematical formulation of a 3D generalized pyrolysis model, Gpyro3D, and its coupling to a pre-release version of FDS6. After presenting verification studies for the pyrolysis model and its coupling to FDS, a 3D simulation of oxidative pyrolysis of thermally irradiated wet wood is presented, and the results are compared to analogous experimental observations. Finally, the fully coupled Gpyro3D/FDS6 model is used to simulate fire development in wood crib.

\section{MATHEMATICAL FORMULATION OF GPYRO3D}

Gpyro3D is the product of continued development of Gpyro, originally developed as a 1D model [9-11], and later extended to 2D [12]. Gpyro has since been extended to three dimensions, coupled to a pre-release version of FDS 6 (SVN 15146), and modified to handle complex geometries (i.e., objects other than parallelpipeds). Currently, assumptions inherent in the formulation of Gpyro3D include:

- $\quad$ Each condensed-phase species has well-defined properties such as bulk density, specific heat capacity, effective thermal conductivity (in the $x, y$, and $z$ directions), permeability (in the $x, y$, and $z$ direction), and porosity.

- Specific heat capacity and directional thermal conductivity vary as $\phi(T)=\phi_{0}\left(T / T_{r}\right)^{n_{\phi}}$, where $T_{r}$ is a reference temperature, $\phi_{0}$ is the value of $\phi$ at $T_{r}$, and $n_{\bullet}$ specifies whether $\phi$ increases or decreases with $T$.

- Density of a particular species does not vary with temperature.

- There is no net shrinkage (volume change) due to reactions or density variation. Gpyro can, for 1D formulations, account for shrinkage/swelling but this has not yet been extended to 3D.

- Radiation heat transfer across pores is accounted for by adding a contribution to the effective thermal conductivity that varies as $\gamma T^{3}$ where $\gamma$ is a user-specified model parameter.

- Average properties in conservation equations are calculated by appropriate mass or volume fraction weighting.

- Pressure-driven flow through porous media follows Darcy's law.

- All gaseous species have equal diffusion coefficients.

- The Schmidt number is unity $(v=D)$. 
To limit the length and complexity of this paper, several simplifications that are not inherent to the formulation of Gpyro3D are invoked, specifically:

- Gas-phase and condensed-phase inside a fuel particle are in thermal equilibrium, i.e., $T=T_{g}$. In general, Gpyro solves separate conservation equations for the gaseous and condensed phases.

- No homogeneous gas-phase reactions occur. Gpyro3D has the capability to simulate homogeneous gas-phase reactions occurring inside the voids of a porous medium.

- Buoyancy effects inside the decomposing solid are negligible. Gpyro3D has the capability to model buoyant flows inside decomposing porous media.

- The solid is opaque to thermal radiation. Gpyro3D can account for in-depth radiation absorption (diathermancy) as well as radiation heat transfer across pores.

Under these inherent assumptions and simplifications specific to this paper, the resultant three-dimensional conservation equations (and auxiliary relations such as Darcy's law and the ideal gas law) that govern heat transfer, mass transfer, and chemical reaction inside a pyrolyzing solid are:

$$
\begin{aligned}
& \frac{\partial \bar{\rho}}{\partial t}=-\dot{\omega}_{f g}^{\prime \prime \prime} \\
& \frac{\partial\left(\bar{\rho} Y_{i}\right)}{\partial t}=\dot{\omega}_{f i}^{\prime \prime \prime}-\dot{\omega}_{d i}^{\prime \prime \prime} \\
& \frac{\partial(\bar{\rho} \bar{h})}{\partial t}+\frac{\partial\left(\dot{m}_{x}^{\prime \prime} h_{g}\right)}{\partial x}+\frac{\partial\left(\dot{m}_{y}^{\prime \prime} h_{g}\right)}{\partial y}+\frac{\partial\left(\dot{m}_{z}^{\prime \prime} h_{g}\right)}{\partial z}=-\frac{\partial \dot{q}_{x}^{\prime \prime}}{\partial x}-\frac{\partial \dot{q}_{y}^{\prime \prime}}{\partial y}-\frac{\partial \dot{q}_{z}^{\prime \prime}}{\partial z}+\dot{Q}_{s}^{\prime \prime \prime}+\sum_{i=1}^{M}\left(\dot{\omega}_{f i}^{\prime \prime \prime}-\dot{\omega}_{d i}^{\prime \prime \prime}\right) h_{i} \\
& \dot{q}_{x}^{\prime \prime}=-\overline{k_{x}} \frac{\partial T}{\partial x} \quad \dot{q}_{y}^{\prime \prime}=-\overline{k_{y}} \frac{\partial T}{\partial y} \quad \dot{q}_{z}^{\prime \prime}=-\overline{k_{z}} \frac{\partial T}{\partial z} \\
& \frac{\partial\left(\rho_{g} \bar{\psi} Y_{j}\right)}{\partial t}+\frac{\partial\left(\dot{m}_{x}^{\prime \prime} Y_{j}\right)}{\partial x}+\frac{\partial\left(\dot{m}_{y}^{\prime \prime} Y_{j}\right)}{\partial y}+\frac{\partial\left(\dot{m}_{z}^{\prime \prime} Y_{j}\right)}{\partial z}=-\frac{\partial \dot{j}_{j, x}^{\prime \prime}}{\partial x}-\frac{\partial \dot{j}_{j, y}^{\prime \prime}}{\partial y}-\frac{\partial j_{j, z}^{\prime \prime}}{\partial z}+\dot{\omega}_{f j}^{\prime \prime \prime}-\dot{\omega}_{d j}^{\prime \prime \prime} \\
& \dot{j}_{j, x}^{\prime \prime}=-\bar{\psi} \rho_{g} D \frac{\partial Y_{j}}{\partial x} \quad \dot{j}_{j, y}^{\prime \prime}=-\bar{\psi} \rho_{g} D \frac{\partial Y_{j}}{\partial y} \\
& \frac{\partial\left(\rho_{g} \bar{\psi}\right)}{\partial t}+\frac{\partial \dot{m}_{x}^{\prime \prime}}{\partial x}+\frac{\partial \dot{m}_{y}^{\prime \prime}}{\partial y}+\frac{\partial \dot{m}_{z}^{\prime \prime}}{\partial z}=\dot{\omega}_{f g}^{\prime \prime \prime} \\
& \dot{m}_{x}^{\prime \prime}=-\frac{K_{x}}{v} \frac{\partial P}{\partial x} \quad \frac{\dot{m}_{g}}{P M} D \frac{\partial Y_{j}}{\partial z} \\
& \rho_{y}^{\prime \prime}=-\frac{\partial P}{v} \frac{\dot{m}_{z}^{\prime \prime}}{\partial y}=-\frac{\frac{K_{z}}{v}}{\partial P} \frac{\partial P}{\partial z}
\end{aligned}
$$

The internal pressure distribution is calculated by substituting Eq. 7 and Eq. 6 into Eq. 5 and solving the resultant pressure evolution equation for the unknown pressure $P$. Pressure-driven mass flux is then calculated from the pressure distribution $(P(x, y, z))$ using Eq. 6 (Darcy's law). In the preceding equations, a subscript $i$ refers to the condensed-phase and a subscript $j$ refers to the gas-phase. An overbar denotes a weighted or averaged quantity, i.e. $\overline{k_{x}}=\sum X_{i} k_{x i}$. Anisotropic materials are simulated through specification of directional thermal conductivities $\left(k_{x}, k_{y}\right.$, and $\left.k_{z}\right)$ and permeabilities $\left(K_{x}, K_{y}\right.$, and $\left.K_{z}\right)$.

\section{Chemical reaction source terms}

The governing equations presented above contain several source terms attributed to chemical reactions $\left(\dot{\omega}_{f i}^{\prime \prime \prime}, \dot{\omega}_{d i}^{\prime \prime \prime}, \dot{\omega}_{f j}^{\prime \prime \prime}, \dot{\omega}_{d j}^{\prime \prime \prime}, \dot{\omega}_{f g}^{\prime \prime \prime}\right.$, and $\left.\dot{Q}_{s}^{\prime \prime \prime}\right)$ that must be quantified. Since the focus of the current paper is 3D 
effects, and these source terms are treated no differently in Gpyro3D than in earlier work [9-11], the reader is referred to this earlier work for full details regarding threat treatment of chemical reaction source terms.

\section{D numerical solution methodology}

The governing equations described above are discretized into grid cells having size $\Delta x$ in the $x$ direction, $\Delta y$ in the $y$ direction, and $\Delta z$ in the $z$ direction. The timestep is $\Delta t$. Due to the nonlinearity introduced by the source terms and temperature-dependent thermophysical properties, a fully-implicit formulation is adopted for solution of all equations. The numerical solution technique follows Patankar [13], yielding a system of coupled algebraic equations of the general form:

$$
a_{p} \phi_{P}=a_{E} \phi_{E}+a_{W} \phi_{W}+a_{s} \phi_{S}+a_{N} \phi_{N}+a_{T} \phi_{T}+a_{B} \phi_{B}+b_{P}
$$

In Eq. 8, $\phi$ is the dependent variable (such as condensed-phase enthalpy, gas-phase species mass fraction, etc.) and the subscripts indicate values of $\phi$ or its associated discretization coefficients (" $a$ " and " $b$ ") in particular cells relative to cell $P$ (the "point", or central, cell in the discretization stencil). The remaining subscripts have the following meaning: $E$ (east) is the closest cell in the $-x$ direction from cell $P$; $W$ (west) is the closest cell in the $+x$ direction; $S$ (south) is the cell in the $-y$ direction; $N$ (north) is the cell in the $+y$ direction; $T$ (top) is the cell in the $-z$ direction; and $B$ (bottom) is the cell in the $+z$ direction. Using the condensed-phase mass conservation equation as an example, the $a$ and $b$ coefficients in Eq. 8 are:

$$
\begin{aligned}
& a_{E}=\frac{\left(\bar{k}_{x} / \bar{c}\right)_{e}}{(\delta x)_{e}} \Delta y \Delta z \quad a_{W}=\frac{\left(\bar{k}_{x} / \bar{c}\right)_{w}}{(\delta x)_{w}} \Delta y \Delta z \\
& a_{S}=\frac{\left(\bar{k}_{y} / \bar{c}\right)_{s}}{(\delta y)_{s}} \Delta x \Delta z \quad a_{N}=\frac{\left(\bar{k}_{y} / \bar{c}\right)_{n}}{(\delta y)_{n}} \Delta x \Delta z \\
& a_{T}=\frac{\left(\bar{k}_{z} / \bar{c}\right)_{t}}{(\delta z)_{t}} \Delta x \Delta y \quad a_{B}=\frac{\left(\bar{k}_{z} / \bar{c}\right)_{b}}{(\delta z)_{b}} \Delta x \Delta y
\end{aligned}
$$

$b_{P}=a_{P}^{\circ} h_{P}^{\circ}+\dot{S}_{P}^{\prime \prime \prime} \Delta x \Delta y \Delta z \quad ; \quad \dot{S}_{P}^{\prime \prime \prime}=\dot{Q}_{s}^{\prime \prime \prime}+\sum_{i=1}^{M}\left(\dot{\omega}_{f i}^{\prime \prime \prime}-\dot{\omega}_{d i}^{\prime \prime \prime}\right) h_{i}-\frac{\partial\left(\dot{m}_{x}^{\prime \prime} h_{g}\right)}{\partial x}-\frac{\partial\left(\dot{m}_{y}^{\prime \prime} h_{g}\right)}{\partial y}-\frac{\partial\left(\dot{m}_{z}^{\prime \prime} h_{g}\right)}{\partial z}$

$a_{p}=a_{E}+a_{W}+a_{S}+a_{N}+a_{T}+a_{B}+a_{P}^{\circ}$ where $a_{P}^{\circ}=\bar{\rho}_{P}^{\circ} \frac{\Delta x \Delta y \Delta z}{\Delta t}$

With regard to the cardinal directions, uppercase subscripts $(E, W, S, N, B, T)$ denote values at cell centers while lowercase subscripts $(e, w, s, n, b, t)$ denote values at the interfaces between cells. All quantities are defined at time $t+\Delta t$, except that quantities with a superscript " $\mathrm{o}$ " are defined at time $t$. The gas-phase species, gas-phase momentum, and condensed-phase energy conservation equations are solved using a tridiagonal matrix algorithm (TDMA) and Gauss Seidel iteration. It is possible that alternate solvers may reduce computational cost. Convective terms are fully upwinded by default, although other schemes are available. The condensed-phase mass and condensed-phase species conservation equations are solved with an implicit solver that uses relaxation to prevent divergence.

\section{Initial conditions}

The discretized forms of Eqs. 1 and 2, condensed-phase mass and species conservation, reduce to ordinary differential equations in each computational cell. Therefore, no boundary conditions are required, only initial conditions that describe the mass fraction of each condensed-phase species are required. Since each condensed-phase species has a well-defined bulk density, the initial density required for solution of Eq. 1 is calculated internally from knowledge of the initial condensed-phase mass fractions. The initial condition on Eq. 3a, condensed-phase energy conservation, is established by specifying the initial temperature, which is then used internally to calculate the initial weighted enthalpy $\bar{h}$. Similarly, the initial conditions on Eq. $4 \mathrm{a}$ (gas-phase species conservation) and the pressure evolution equation are established by specifying the initial gas-phase species mass fraction and the initial pressure, respectively. 


\section{Boundary conditions}

Two general types of boundary conditions are implemented in Gpyro: Fixed value, and fixed flux. For fixed value boundary conditions, the $\phi$ value of the boundary cell is set to the desired fixed value by setting, in Eq. 8, $a_{P}$ to $1, b_{P}$ to the desired $\phi$ value, and all remaining $a$ coefficients to 0 . Fixed flux boundary conditions are more complicated to implement. For illustrative purposes, the condensed-phase energy boundary condition in the $x$ direction (at $x=0$ ) will be used to demonstrate how boundary conditions are implemented numerically. The condensed-phase energy equation $x$-direction boundary condition for a face located at $x=0$, with $\dot{q}_{r, x}^{\prime \prime}$ as the $x$-direction incident radiant heat flux, is:

$$
-\left.\bar{k}_{x} \frac{\partial T}{\partial x}\right|_{x=0}=-\left.\frac{\bar{k}}{\bar{c}} \frac{\partial \bar{h}}{\partial x}\right|_{x=0}=\bar{\varepsilon} \dot{q}_{r, x}^{\prime \prime}-\bar{\varepsilon} \sigma\left(\left.T^{4}\right|_{x=0}-T_{\infty}^{4}\right)-h_{c}\left(\left.T\right|_{x=0}-T_{\infty}\right)=\dot{q}_{n e t}^{\prime \prime}
$$

Eq. 13, after discretization to cast it in the form of Eq. 8, becomes:

$$
-\left(\frac{\bar{k}_{x}}{\bar{c}}\right)_{e} \frac{\bar{h}_{E}-\bar{h}_{P}}{(\delta x)_{e}}=\dot{q}_{n e t}^{\prime \prime}
$$

After multiplying both sides by of Eq. 14 by $\Delta y \Delta z$ and substituting the definition of $a_{E}$ from Eq. 9a:

$$
-\frac{\left(\bar{k}_{x} / \bar{c}\right)_{e}}{(\delta x)_{e}} \Delta y \Delta z\left(\bar{h}_{E}-\bar{h}_{P}\right)=\dot{q}_{n e t}^{\prime \prime} \Delta y \Delta z \Rightarrow-a_{E}\left(\bar{h}_{E}-\bar{h}_{P}\right)=\dot{q}_{n e t}^{\prime \prime} \Delta y \Delta z
$$

Eq. 15 can be rewritten as:

$a_{E}\left(\bar{h}_{P}-\bar{h}_{E}\right)=\dot{S}_{b c}^{\prime \prime \prime} \Delta x \Delta y \Delta z \quad$ where $\quad \dot{S}_{b c}^{\prime \prime \prime}=\frac{\dot{q}_{n e t}^{\prime \prime}}{\Delta x}$

Eq. 16 illustrates the way that all fixed flux boundary conditions are implemented in Gpyro3D. Essentially, the flux at the boundary is first converted to a source term by dividing that flux by the grid spacing in the direction parallel to the flux. That source term is then added to $\dot{S}_{P}^{\prime \prime \prime}$ in Eq. 11 (or the analogous source term in the other conservation equations). Mathematically, this approach is equivalent to relating the $\phi$ gradient at the boundary to an appropriate flux (i.e., Eq. 13), but it is much easier to implement numerically.

The "boundary condition source terms", for brevity written only for faces pointing in the $-x$ direction (and assumed to be at $x=0$ ), are listed in Table 1. Although radiative heat losses were not considered from the "back" face in earlier 1D implementations of Gpyro, in the current 3D formulation the solid energy equation explicitly includes convective and radiative losses at all boundaries, regardless of orientation. Note that in Table $1, h_{m}$ is a mass transfer coefficient.

Table 1. Boundary condition source terms.

\begin{tabular}{|l|l|}
\hline Conservation Equation & $\boldsymbol{x}$-direction boundary condition source term (at $\boldsymbol{x}=\mathbf{0})$ \\
\hline Solid energy (Eq. 3) & $\dot{S}_{b c}^{\prime \prime \prime} \Delta x=\bar{\varepsilon} \dot{q}_{r, x}^{\prime \prime}-\bar{\varepsilon} \sigma\left(\left.T^{4}\right|_{x=0}-T_{\infty}^{4}\right)-h_{c}\left(\left.T\right|_{x=0}-T_{\infty}\right)$ \\
\hline Gas species (Eq. 4) & $\dot{S}_{b c}^{\prime \prime \prime} \Delta x=\left(h_{m}+\max \left(\left.\dot{m}_{x}^{\prime \prime}\right|_{x=0}, 0\right)\right)\left(Y_{j, \infty}-\left.Y_{j}\right|_{x=0}\right)$ \\
\hline Pressure evolution equation & $\dot{S}_{b c}^{\prime \prime \prime} \Delta x=\dot{m}_{x}^{\prime \prime}$ \\
\hline
\end{tabular}

For a decomposing solid, it is almost always the case that $\left.\dot{m}_{x}^{\prime \prime}\right|_{x=0}<0$, meaning that gases convect out of the decomposing solid, driven by a pressure gradient that forms when condensed-phase mass is converted to gas-phase mass. Consequently, the term $\max \left(\left.\dot{m}_{x}^{\prime \prime}\right|_{x=0}, 0\right)$ in the gas species boundary condition usually evaluates to 0 ; however, when the pressure gradient is such that gases are convecting into the solid, $\left.\dot{m}_{x}^{\prime \prime}\right|_{x=0}>0$. This may be important for situations where a forced flow is introduced into porous media. 


\section{Complex geometry}

Similar to the way that geometry is specified in FDS, geometrically complex objects are represented in Gpyro3D through specification of a regular Cartesian mesh and multiple parallelepipeds that build up the geometry block by block. A file containing \&OBST (obstruction) namelist groups of same form used to specify geometry in FDS is parsed to mask cells within the Gpyro3D computational domain that represent the solid-phase geometry. After the geometry is read in, cells that require boundary conditions are identified. Thus, "boundary" conditions may be applied at interior grid points that do not actually correspond to the boundary of the computational domain, which is a parallelepiped. For grid cells that are not marked as being part of the solid-phase geometry, the $a_{p}$ coefficient is set to 1 , the $b_{p}$ coefficient is set to its initial value, and all other discretization coefficients are set to 0 . In this way, an efficient block tridiagonal solver can be used. The output routines check the geometry mask so that outputs in areas that are not marked as being part of the geometry will not be reported, as the numerical solution in areas that are not part of the geometry is meaningless. A complex geometry example will be demonstrated later.

\section{Coupling to FDS and other Gpyro3D domains}

Three types of boundary/interface conditions are handled in Gpyro: 1) User-specified value or flux at a boundary, 2) Heat, species, and mass exchange at the interface between a Gpyro region and an FDS region, and 3) Heat, species, and mass exchange at the interface between two Gpyro regions. Of these, only 1) has been addressed earlier in this paper.

Gpyro is coupled to FDS using the same internal linkages that FDS uses to link its 1D pyrolysis model to the gas-phase solver. These linkages are described in Section 3.2.3 of the FDS6 Technical Reference [1]. Gpyro3D obtains the radiant and convective heat flux from FDS. This is applied as a boundary condition using the methodology described above, and the solid-phase solution is advanced forward in time. The updated surface temperature is then passed from Gpyro3D to FDS. An analogous procedure is followed for gas-phase species, with the calculated mass flux of each species being passed from Gpyro3D to FDS. Since it is often desirable to use a finer grid spacing in the condensed-phase than in the gas-phase, the coupling between Gpyro3D and FDS is implemented in a way that allows one FDS face to be mapped to multiple Gpyro3D faces. For example, if a grid spacing of $3 \mathrm{~mm}$ is used in the gas phase and a grid spacing of $1 \mathrm{~mm}$ is used in the condensed phase, then each FDS face would "see" nine Gpyro3D faces, but each of the nine Gpyro3D faces would "see" only one FDS face. When the grid spacing is not the same in the Gpyro3D and FDS, area-averaged quantities (temperature, species mass flux, etc.) are passed to FDS.

Finally, a Gpyro3D region may abut another Gpyro3D region. For example, in a wood crib, individual wood elements touch other wood elements. For analogous situations, it is assumed that Gpyro3D regions are in perfect thermal contact. The temperature gradient at each face is used to calculate a heat flux; the heat flux leaving the hotter face is of the same magnitude as the heat flux entering the colder face. Currently, mass and momentum are not transferred between Gpyro3D regions.

\section{MODEL VERIFICATION}

The author has previously subjected Gpyro to several verification exercises, some of which are reported in Ref. [9]. Collectively, these verification exercises indicate that, when the spatial and temporal discretization is such that the numerical solution becomes grid-independent, numerical solutions match analogous exact solutions to within $<0.5 \%$. Since the majority of this was done before Gpyro was extended to $3 \mathrm{D}$ and coupled to FDS6, this section addresses verification of Gpyro3D and its coupling to FDS6.

\section{Transient three dimensional heat conduction in a parallelepiped}

Consider a constant-property solid parallelepiped initially at temperature $T_{0}$ having dimensions $0<x<a, 0$ $<y<b$, and $0<z<c$. It is insulated at the faces corresponding to $x=0, y=0$, and $z=0$ and it loses heat to the ambient at zero temperature by Newtonian cooling proportional to heat transfer coefficients $h_{a}, h_{b}$, and $h_{c}$ at $x=a, y=b$, and $z=c$, respectively. The mathematical statement of this heat conduction problem is:

$$
\frac{\partial^{2} T}{\partial x^{2}}+\frac{\partial^{2} T}{\partial y^{2}}+\frac{\partial^{2} T}{\partial z^{2}}=\frac{1}{\alpha} \frac{\partial T}{\partial t} \text { with }\left.T\right|_{t=0}=T_{0}
$$


$\left.\frac{\partial T}{\partial x}\right|_{x=0}=0 ;-\left.k \frac{\partial T}{\partial x}\right|_{x=a}=h_{a} T ;\left.\frac{\partial T}{\partial y}\right|_{y=0}=0 ;-\left.k \frac{\partial T}{\partial y}\right|_{y=b}=h_{b} T ;\left.\quad \frac{\partial T}{\partial z}\right|_{z=0}=0 ;-\left.k \frac{\partial T}{\partial z}\right|_{z=c}=h_{c} T$

Note that radiative heat losses are not included. The exact solution, derived via separation of variables, is:

$$
\begin{aligned}
& T=\sum_{\ell=1}^{\infty} \sum_{m=1}^{\infty} \sum_{n=1}^{\infty} C_{\ell m n} \cos \left(\beta_{\ell} x\right) \cos \left(\gamma_{m} y\right) \cos \left(\delta_{n} z\right) \exp \left(-\left(\beta_{\ell}^{2}+\gamma_{m}^{2}+\delta_{n}^{2}\right) a t\right) \\
& C_{\ell m n}=64 T_{0} \frac{\sin \left(\beta_{\ell} a\right) \sin \left(\gamma_{m} b\right) \sin \left(\delta_{n} c\right)}{\left(2 \beta_{\ell} a+\sin \left(2 \beta_{\ell} a\right)\right)\left(2 \gamma_{m} b+\sin \left(2 \gamma_{m} b\right)\right)\left(2 \delta_{n} c+\sin \left(2 \delta_{n} c\right)\right)}
\end{aligned}
$$

where the eigenvalues of the problem are obtained from the relations:

$$
\cot \left(\beta_{\ell} a\right)=\frac{k}{h_{a}} \beta_{\ell} ; \cot \left(\gamma_{m} b\right)=\frac{k}{h_{b}} \gamma_{m} ; \cot \left(\delta_{n} c\right)=\frac{k}{h_{c}} \gamma_{n}
$$

The exact solution was calculated by summing over the first $300 x, y$, and $z$ eigenvalues with $a=b=c=$ $0.025 \mathrm{~m}, h_{a}=h_{b}=h_{c}=10 \mathrm{~W} / \mathrm{m}^{2}-\mathrm{K}, T_{0}=1000 \mathrm{~K}, k=1 \mathrm{~W} / \mathrm{m}-\mathrm{K}$, and $\alpha=1 \times 10^{-6} \mathrm{~m}^{2} / \mathrm{s}$. The exact solution was compared to the analogous Gpyro3D calculation using a grid spacing of $0.5 \mathrm{~mm}$ and a timestep of 0.05 s. Surface reradiation losses were excluded by setting a flag in Gpyro3D that forces the term $\bar{\varepsilon} \sigma\left(\left.T^{4}\right|_{x=0}-T_{\infty}^{4}\right)$ in Eq. 13 to 0 . The numerical error was calculated as $E=100 \times\left(T_{g}-T_{e}\right) /\left(T_{0}-T_{e}\right)$ where $T_{g}$ is the temperature calculated with Gpyro3D and $T_{e}$ is temperature from the exact solution. Figure 1 shows temperature profiles at $z=0.012$ (left panels) and numerical error (right panels) at two times. The solution was found to match within approximately $0.5 \%$.
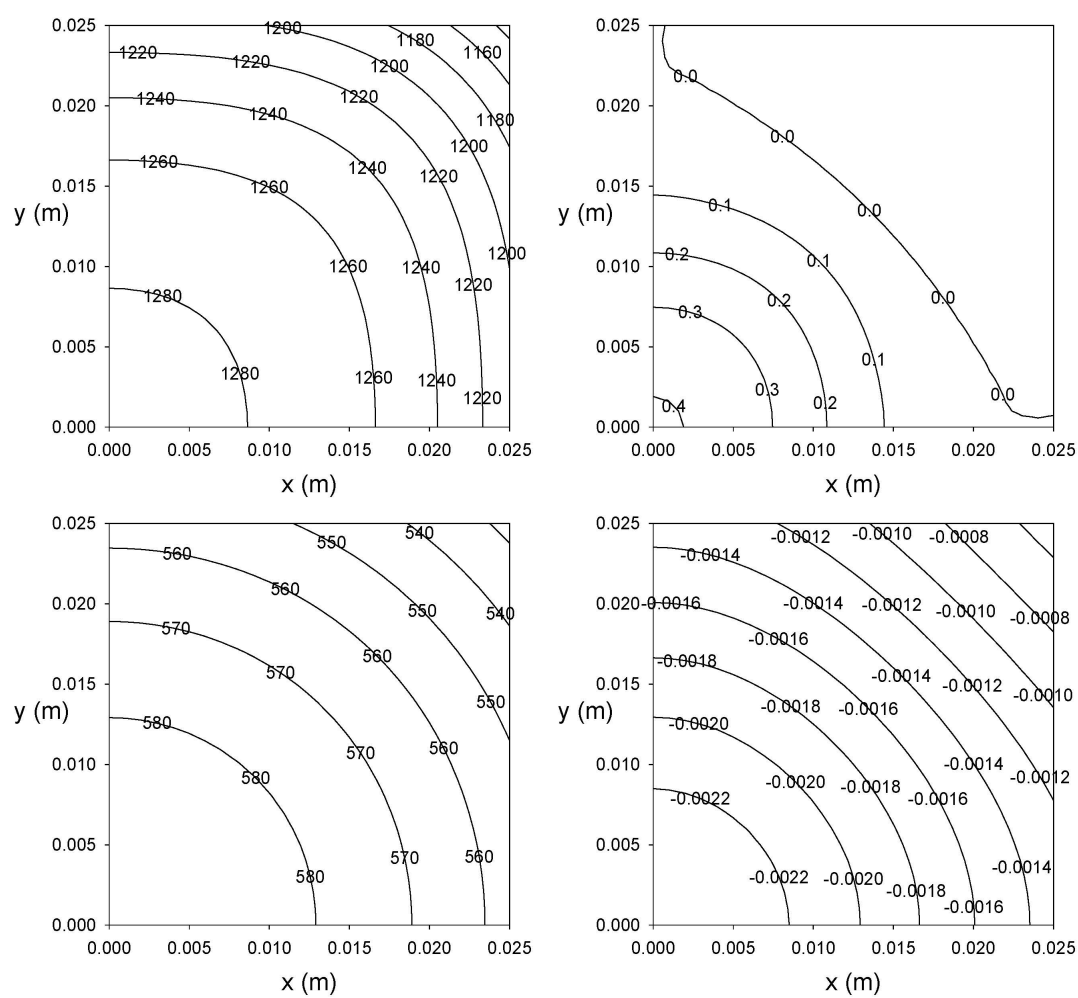

Figure 1. Graphical solution to Eq. 17 in a plane corresponding to $z=0.012$. Left panes: exact solution; Right panes: percent difference between exact and Gpyro3D solution. Top panes: 60 s. Bottom: 1200s. 


\section{Transient three dimensional heat conduction with internal heat generation in a complex geometry}

Consider a solid sphere with radius $a$ that experiences internal volumetric heat generation at a rate $g_{0}$ $\left(\mathrm{W} / \mathrm{m}^{3}\right)$. For initial temperature $T_{0}$ and boundary at $r=a$ held at temperature $T_{0}$ the mathematical statement of the heat conduction problem is:

$$
\begin{gathered}
\frac{1}{\alpha} \frac{\partial T}{\partial t}=\frac{1}{r^{2}} \frac{\partial}{\partial r}\left(\frac{\partial T}{\partial r}\right)+\frac{g_{0}}{k} \text { with }\left.T\right|_{t=0}=T_{0} \\
\left.T\right|_{r=a}=T_{0} \quad ;\left.\quad \frac{\partial T}{\partial r}\right|_{r=0}=0
\end{gathered}
$$

The exact solution to Eq. 20 is:

$$
T-T_{0}=\frac{g_{0}}{6 k}\left(a^{2}-r^{2}\right)+\frac{2 g_{0} a^{3}}{k \pi^{3} r} \sum_{n=1}^{\infty} \frac{(-1)^{n}}{n^{3}} \sin \left(\frac{n \pi r}{a}\right) \exp \left(-\alpha t \frac{n^{2} \pi^{2}}{a^{2}}\right)
$$

Gpyro3D was used to conduct a numerical simulation analogous to this exact solution for a sphere with radius 0.025 centered at $(x=0, y=0, z=0)$, internal heat generated at a rate of $200 \mathrm{~kW} / \mathrm{m}^{3}, k=1 \mathrm{~W} / \mathrm{m}-\mathrm{K}, \rho=$ $1000 \mathrm{~kg} / \mathrm{m}^{3}$, and $c=1 \mathrm{~kJ} / \mathrm{kg}-\mathrm{K}$. The sphere geometry was specified on a uniform Cartesian grid with resolution $0.5 \mathrm{~mm}$ using the masking procedure described earlier. Calculated temperature contours in $x y$ and $x z$ planes through "stair stepped" geometry are shown in Figure 2a. The numerical solution for $0<x<$ $a$ at $y=0$ and $z=0$ (essentially, the radius of the sphere) is compared to the exact solution in Figure $2 \mathrm{~b}$ at several times. It is seen that the solutions match very closely. The minor difference between the exact and numerical solution at later times is likely due to geometrical differences between a stair stepped sphere (numerical solution) and a true sphere (exact solution).
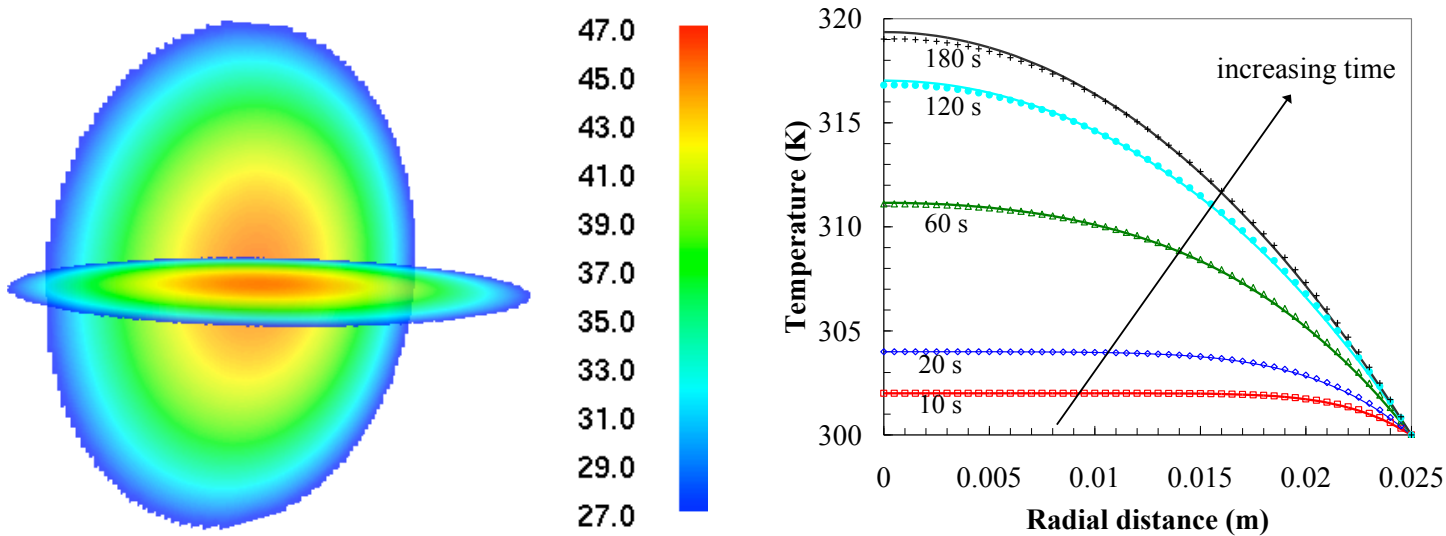

Figure 2. Gpyro3D simulation of transient heat conduction in a sphere with internal heat generation. (a) Temperature contours $\left({ }^{\circ} \mathrm{C}\right)$. (b) Comparison of numerical and exact solution $(\mathrm{K})$.

\section{Gpyro3D to FDS coupling}

As described earlier, Gpyro is coupled to the FDS gas phase using the same basic heat and mass transfer linkages that couple FDS's native 1D pyrolysis model to the gas phase. To assess this coupling, a validation exercise was conducted wherein a Gpyro region was coupled to FDS. The FDS computational domain, shown in Figure $3 \mathrm{a}$, is a sealed cube $0.32 \mathrm{~m}$ on edge with adiabatic boundaries, containing air initially at $300 \mathrm{~K}$ and $1 \mathrm{~atm}$ as well as a cubic Gpyro3D domain $0.1 \mathrm{~m}$ on edge initially at $400 \mathrm{~K}$. Relevant solid properties inside the Gpyro region include $c=1 \mathrm{~kJ} / \mathrm{kg}-\mathrm{K}$ and $\rho=10 \mathrm{~kg} / \mathrm{m}^{3}$. As $t \rightarrow \infty$, the system approaches thermal equilibrium, and that equilibrium temperature can be calculated from a heat balance (the controlling material properties are the ratio of the solid and gas density, volume, and heat capacity) as: 
$T_{e q}=\frac{\rho_{s} V_{s} c_{s} T_{s 0}+\rho_{a} V_{a} c_{v a} T_{a 0}}{\rho_{s} V_{s} c_{s}+\rho_{a} V_{a} c_{v a}}=\frac{T_{a 0}+\frac{(\rho V c)_{s}}{\left(\rho V c_{v}\right)_{a}} T_{s 0}}{1+\frac{(\rho V c)_{s}}{\left(\rho V c_{v}\right)_{a}}}=\frac{300+0.372 \times 400}{1+0.372}=327.1 \mathrm{~K}=54.95^{\circ} \mathrm{C}$

$\frac{(\rho V c)_{s}}{\left(\rho V c_{v}\right)_{a}}=\frac{10 \times 0.1^{3} \times 1}{1.166 \times\left(0.32^{3}-0.1^{3}\right) \times 1.016 / 1.4}=0.372$

At long times, the system becomes nearly homogeneous and the temperatures approach an equilibrium value of $53.5{ }^{\circ} \mathrm{C}$, within $0.45{ }^{\circ} \mathrm{C}$ of the calculated value. The convergence of the temperature at eight locations within the FDS gas-phase to $53.5^{\circ} \mathrm{C}$ is shown in Figure $3 \mathrm{~b}$.

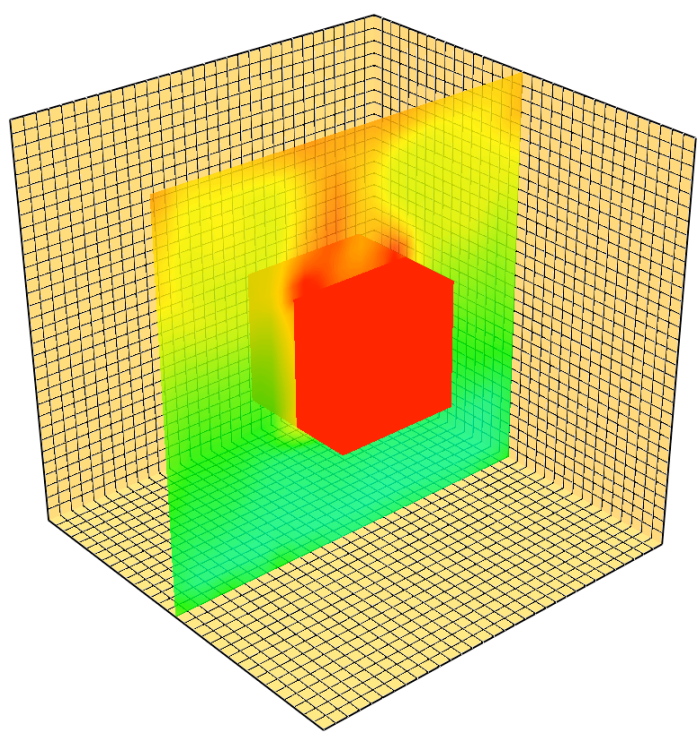

(a)

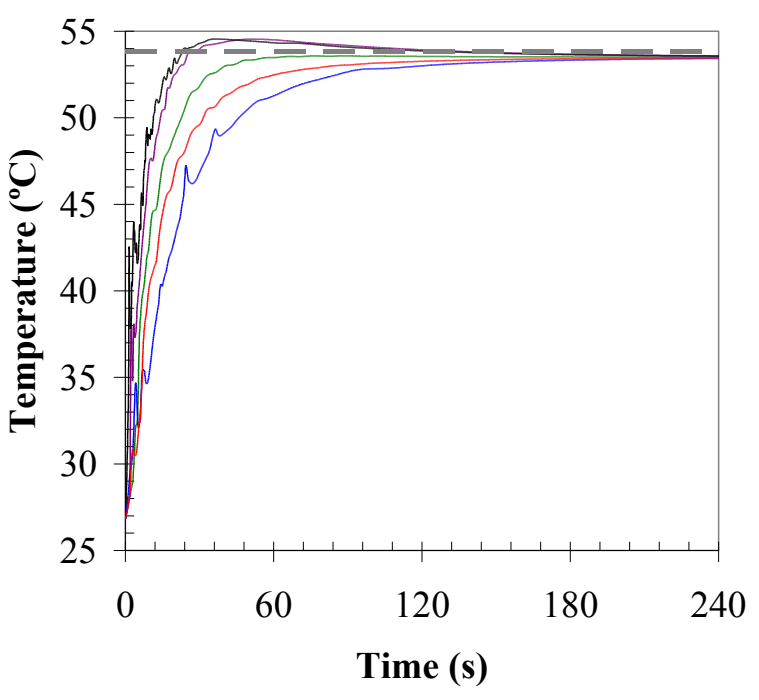

(b)

Figure 3. Gpyro3D to FDS linkage verification exercise. (a) Computational geometry showing temperature contours. (b) Transient temperature at five locations. Dashed location is $54.95{ }^{\circ} \mathrm{C}$.

\section{MODEL VALIDATION}

\section{Three dimensional oxidative pyrolysis of wet wood}

Most bench-scale pyrolysis experiments that have been conducted to date are designed to provide nearly one-dimensional behavior. For this reason, there is currently a dearth of experimental data in the literature appropriate for quantitative validation of a 3D pyrolysis model. However, Reszka [14] conducted a set of wood pyrolysis (and ignition) experiments that exhibit three dimensional effects. A qualitative comparison between one of these experiments and an analogous Gpyro simulation is used here to qualitatively assess the predictive capabilities of Gpyro3D.

The experimental configuration, shown in Figure 4, involved irradiating wood blocks having dimensions of $0.067 \mathrm{~m}$ by $0.10 \mathrm{~m}$ by $0.10 \mathrm{~m}$. Referring to Figure 4 , the left $(-x)$ face was irradiated with a cone heater, while the right $(+x)$ face abutted an aluminum block having dimensions $0.033 \mathrm{~m}$ by $0.10 \mathrm{~m}$ by $0.10 \mathrm{~m}$. The bottom of the wood and aluminum blocks $(-z)$ rested on an insulating ceramic fiberboard, while the top $(+z)$, front $(-y)$, and rear sides $(+y)$ were exposed to the ambient. 


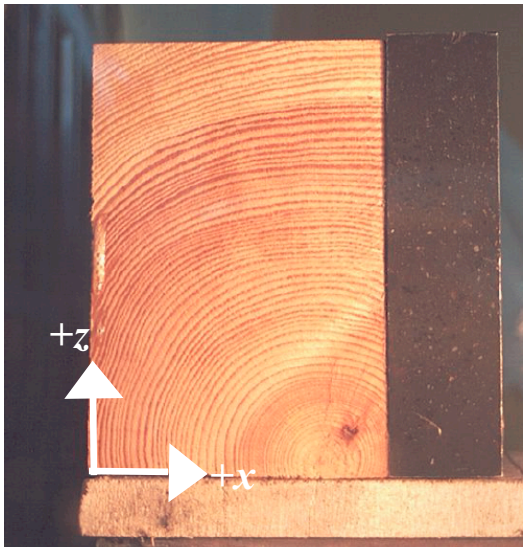

(a)

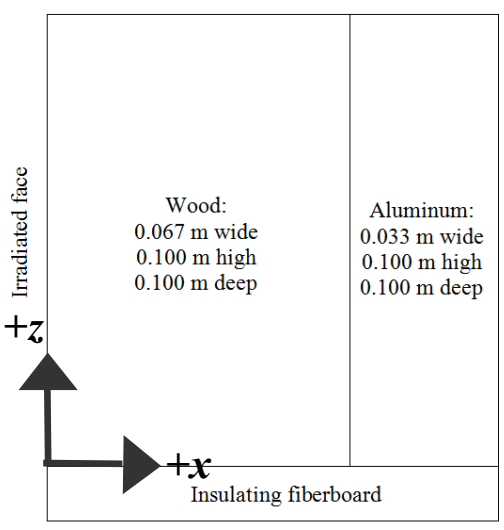

(b)

Figure 4. Reszka [14] pyrolysis experiments. Note that $+y$ direction is into the page.

(a) Photograph of sample assembly. (b) simplified schematic.

Reszka varied the applied heat flux level from $\sim 10$ to $\sim 60 \mathrm{~kW} / \mathrm{m}^{2}$; here a simulation of his experiment at $40 \mathrm{~kW} / \mathrm{m}^{2}$ applied heat flux level is conducted. The wood thermal properties and reaction mechanism are based on earlier work [11] that used a four-step reaction mechanism (conversion of wet wood to dry wood, thermal and oxidative conversion of dry wood to char, and char oxidation to form ash) to investigate oxidative pyrolysis of wood with a 1D simulation. For simplification, thermal conductivity is isotropic.

Figure 5a shows a photograph of Reszka's experiment [14] $1460 \mathrm{~s}$ after the onset of irradiation. An ash layer has formed near the surface, and some erosion or shrinkage has occurred. The modeled ash mass fraction shown in Figure $5 \mathrm{~b}$ (an $x z$ plane through the center of the sample) shows an ash layer that is slightly thinner than that seen in the experiment, most likely because erosion or shrinkage are not included in the model. To the right of the ash layer, in Figure 5c, is a thin char layer. Moving farther to the right, the moisture evaporation front can be seen (Figure 5d). The "hook" that forms in the species and reaction rate profiles is an artifact of the way that the boundary conditions are specified in the simulation compared to the actual boundary conditions in the experiment. In the model, the left face is exposed to a uniform and constant heat flux, while the top face experiences convective and radiative cooling but has no externally applied heat flux. However, in the experiment, there is a nonzero view factor from the cone heater to the top face, and the hook that is seen in the model does not form. 

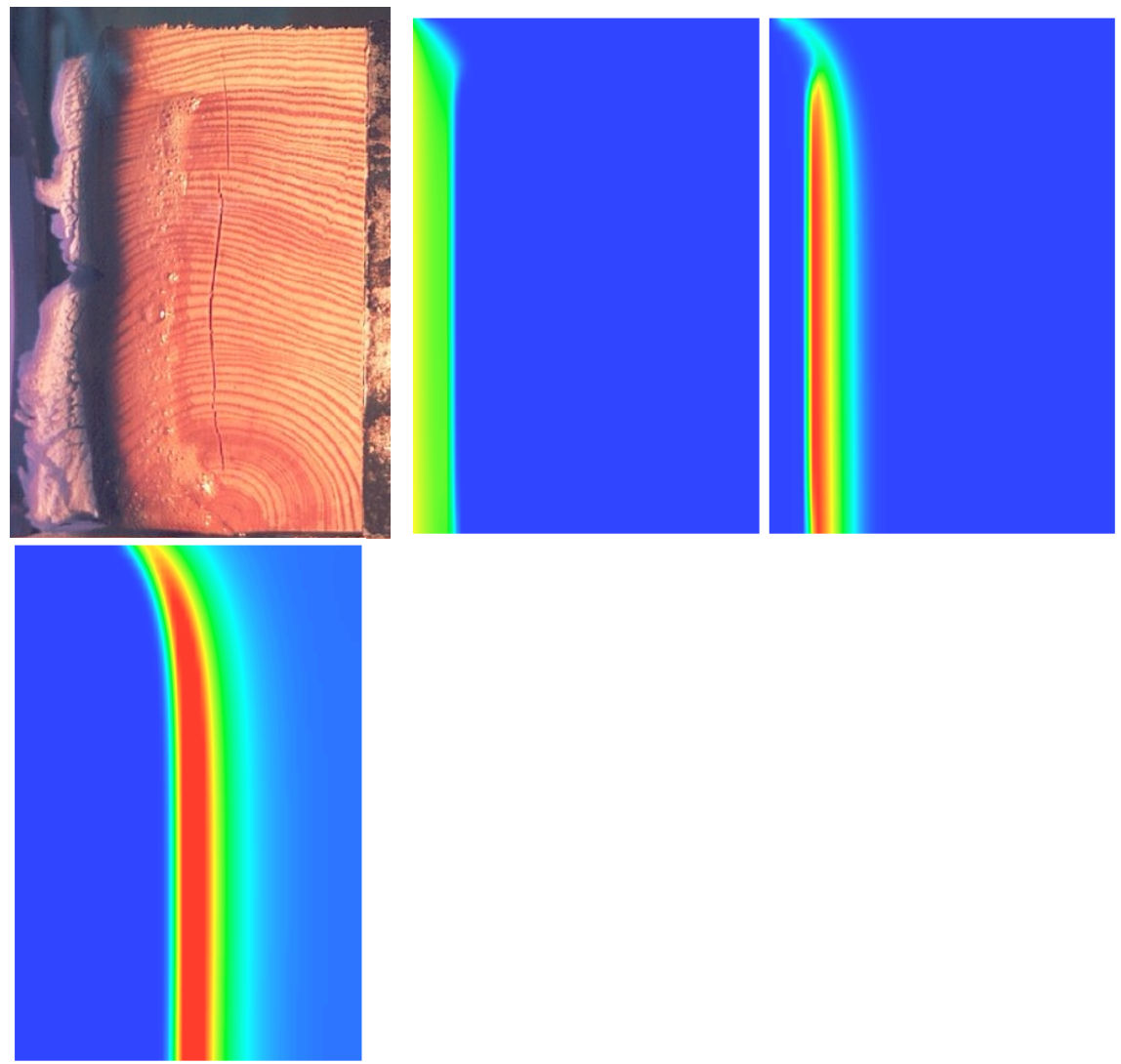

(a)

(b)

(c)

(d)

Figure 5. Comparison of Reszka [14] $40 \mathrm{~kW} / \mathrm{m}^{2}$ experiment @ $1460 \mathrm{~s}$ to Gpyro3D ( $x z$ plane through center of sample). (a) Experiment. (b) Ash mass fraction. (c) Char mass fraction (d) Drying reaction rate.

Figure 6 provides a series of graphics analogous to Figure 5, but 3950s after the onset of irradiation. It appears that the char layer in the model has not propagated as deep into the wood block as in the experiment, again most likely because erosion or surface regression is not considered in the model. The qualitative features of the experiment are however well captured.

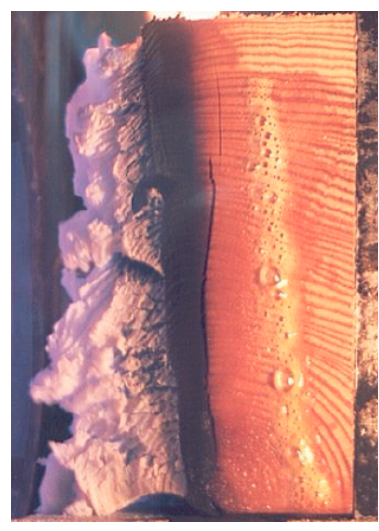

(a)

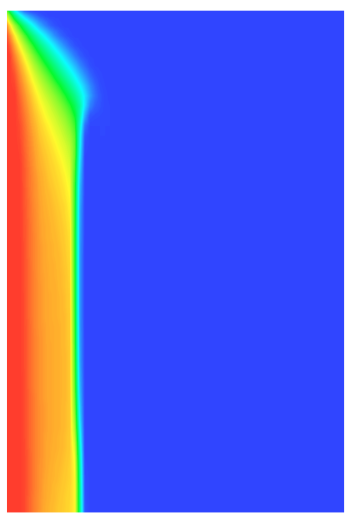

(b)

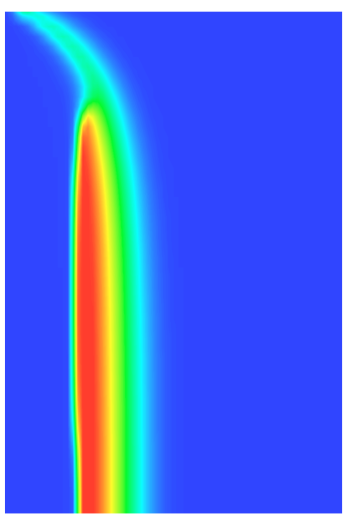

(c)

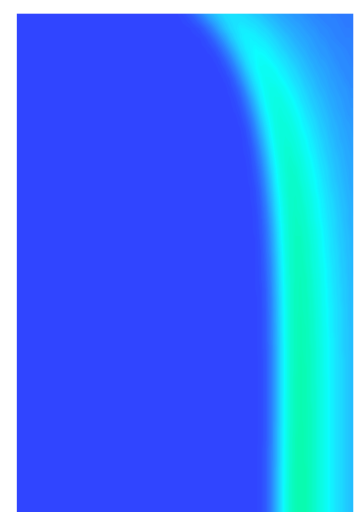

(d)

Figure 6. Comparison of Reszka [14] 40 kW/m² experiment @3950 s to Gpyro3D ( $x z$ plane through center of sample) (a) Experiment. (b) Ash mass fraction. (c) Char mass fraction (d) Drying reaction rate.

The preceding Figures show an $x z$ plane through the center of the wood sample. Figure 7 shows the modeled char mass fraction in an $x y$ (i.e., into the page in Figure 6) plane through the center of the sample at $600 \mathrm{~s}$ and $3600 \mathrm{~s}$. Due to the way the boundary conditions are specified in the simulation, the left and right faces in Figure 7 experience convective and radiative cooling, but no radiative heating, whereas in the 
experiment there is a nonzero view factor between the Cone heater and these sides so they experience radiant heating. This explains the curved nature of the char species profile in Figure 7.

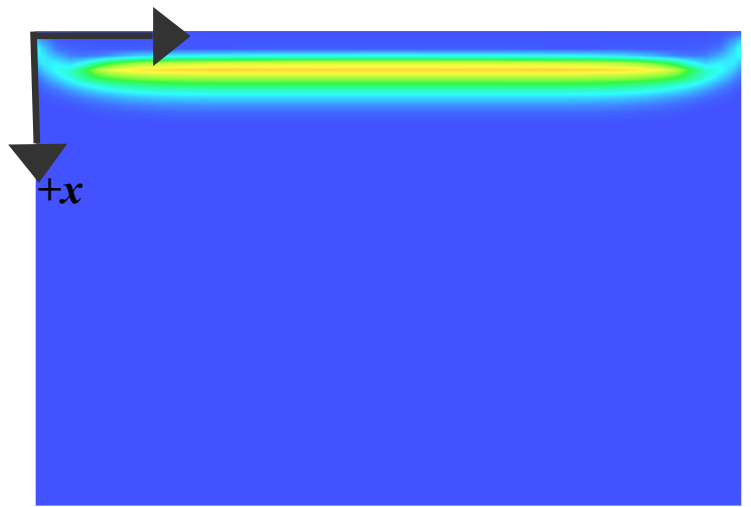

(a)

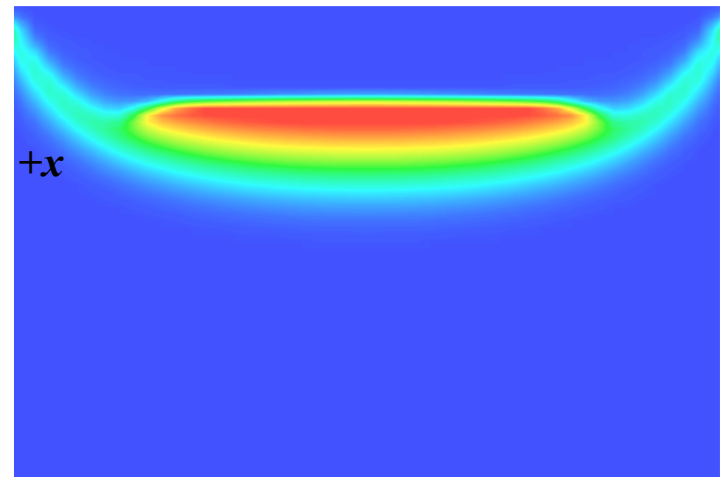

(b)

Figure 7. Modeled char mass fraction in $x y$ plane through center of sample for Reszka [14] $40 \mathrm{~kW} / \mathrm{m}^{2}$ experiment. (a) $600 \mathrm{~s}$. (b) $3600 \mathrm{~s}$.

Two important simplifications inherent to the preceding simulation of Reszka's [14] experiment are: 1) Surface regression is not accounted for, and 2) Thermal conductivity and permeability are isotropic, meaning independent of wood grain orientation. The first simplification is actually a limitation of the current Gpyro3D model formulation (see Concluding Remarks section for additional discussion). The second simplification (isotropic properties) is not a limitation of the current Gpyro3D formulation, which permits directionally dependent thermal conductivity and permeability, but was invoked to simplify the simulation which is intended primarily to qualitatively demonstrate $3 \mathrm{D}$ effects during heating and pyrolysis. Anisotropic properties could be implemented as part of future work.

\section{Fire development in a wood crib}

Pyrolysis modeling studies, which are ostensibly motivated by the desire to predict actual fire development, have almost always used a one-dimensional representation of the condensed-phase. CFD models such as FDS and FireFOAM divide the surface of 3D objects into multiple 1D "patches"; heat is transferred only in the direction normal to the surface of a patch, but not laterally in the directions parallel to the surface to adjacent patches. This 1D description is appropriate for objects where the characteristic length along one coordinate axis is small in comparison to the characteristic length along the other coordinate axes. Wall, ceiling, and floor linings, as well as similarly "thin" objects can be appropriately modeled with a 1D patchwise approach. However, if the characteristic lengths along two coordinate axes are small in comparison to the characteristic length along the third coordinate axis, the validity of simulating an object with a 1D pyrolysis model becomes questionable. To investigate $3 \mathrm{D}$ effects during fire development on fuel elements having two similar characteristic lengths, i.e. where multidimensional heat and mass transfer effects are significant, a fully coupled Gpyro3D/FDS fire growth simulation of a wood crib is presented here.

Simulation geometry and coordinate axes are depicted in Figure 8. The FDS computational domain is 0.48 $\mathrm{m}$ wide $(x)$ by $0.48 \mathrm{~m}$ deep $(y)$ by $0.96 \mathrm{~m}$ high $(z)$. A grid spacing of $0.01 \mathrm{~m}$ is used in the gas-phase (225,792 gas-phase computational cells). A wooden crib is centered in the gas-phase domain such that its base is 0.10 above the bottom of the computational domain. The crib is made up 50 wooden elements with a cross section of $0.02 \mathrm{~m}$ by $0.02 \mathrm{~m}$. There are ten tiers with five elements per tier and a horizontal spacing between elements of $0.04 \mathrm{~m}$. Each element is simulated as a separate Gpyro3D region with a grid spacing of $1 \mathrm{~mm}$ in the two shorter dimensions and $5 \mathrm{~mm}$ in the longer dimension (52,000 Gpyro3D grid cells per element; 2,600,000 Gpyro grid cells in the entire simulation). Each Gpyro region (i.e., each wood element) is coupled to FDS as well as to any Gpyro3D elements to which it abuts. A square ignition burner $0.3 \mathrm{~m}$ on edge, extending $0.05 \mathrm{~m}$ above the bottom of the computational domain, is centered directly below the wooden crib. The ignition burner is fired at $200 \mathrm{~kW} / \mathrm{m}^{2}$ (total heat release rate of $26 \mathrm{~kW}$ ) for $20 \mathrm{~s}$ and then extinguished. Wood material properties are based on previous work [11]. 
Fire development from $30 \mathrm{~s}$ ( $10 \mathrm{~s}$ after the ignition burner was extinguished) to $90 \mathrm{~s}$ is shown qualitatively in Figure 8. During this time, fire progressively spreads to the outer faces of the crib.

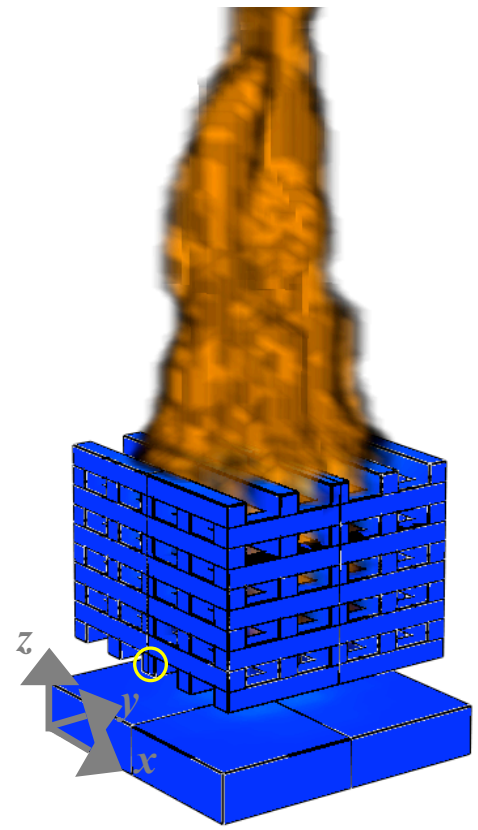

(a)

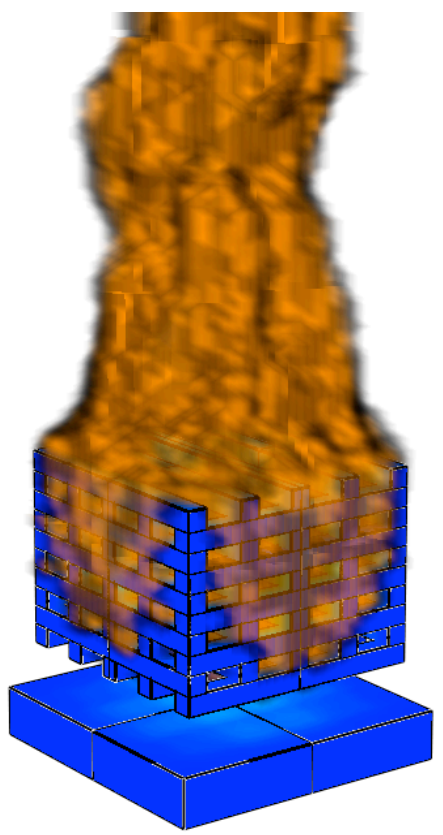

(b)

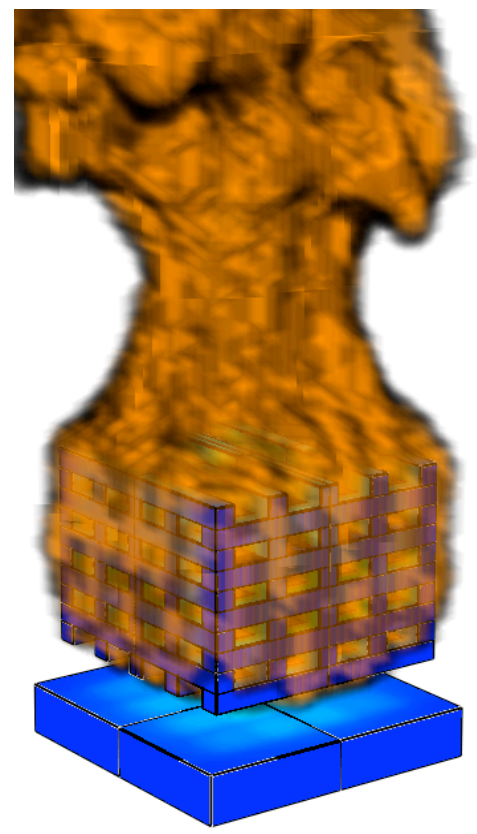

(c)

Figure 8. Crib fire gas-phase reaction zone (flames). (a) 30 s. (b) 60 s. (c) 69 s.

Coordinate system and center wooden element of bottom row identified in panel (a).

Condensed-phase temperature contours in the center wooden element of the bottom row of the crib (circled in Figure 8a) are shown in Figure 9 and Figure 10. Note that the "long" dimension of this element runs in the $+y$ direction. Contours in a vertical $(y z)$ plane through this element are presented in Figure 9. Analogous temperature contours are presented for a horizontal $(x y)$ plane through this element in Figure 10. As time progresses, temperature gradients due to heating at one face begin to merge with temperature gradients attributed to heating from another face. Similarly, areas that are initially "protected" by wooden elements located in the next tier above eventually begin to increase in temperature due to heat conduction in the direction parallel to the exposed face. These phenomena cannot be captured by a 1D patchwise pyrolysis modeling approach.

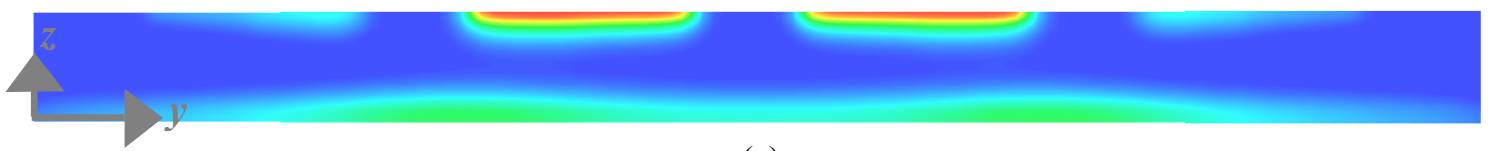

(a)

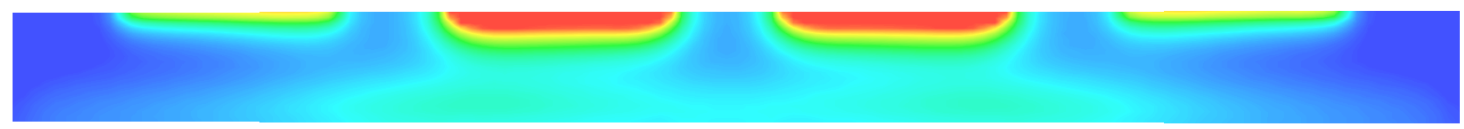

(b)

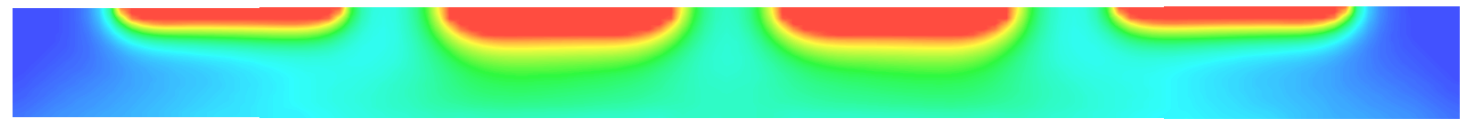

(c)

Figure 9. Temperature contours in a vertical $(y z)$ plane through center wood element in bottom row of crib. (a) $30 \mathrm{~s}$. (b) $60 \mathrm{~s}$. (c) $90 \mathrm{~s}$. Blue $=25^{\circ} \mathrm{C}$. Red $=400^{\circ} \mathrm{C}$ or higher. 
(a)

(b)

(c)

Figure 10. Temperature contours in a horizontal $(x y)$ plane through center wood element in bottom row of crib. (a) $30 \mathrm{~s}$. (b) $60 \mathrm{~s}$. (c) $90 \mathrm{~s}$. Blue $=25^{\circ} \mathrm{C}$. Red $=400{ }^{\circ} \mathrm{C}$ or higher.

\section{CONCLUDING REMARKS}

Gpyro3D, an open source [15] three dimensional generalized pyrolysis model, has been formulated, verified, and used to investigate the pyrolysis of objects in which three dimensional effects are significant, both as a standalone simulation and in a fire growth simulation coupled to FDS. Although this work represents incremental progress in the area of pyrolysis modeling for fire applications, it may be one of the first studies to formulate a 3D generalized pyrolysis model, develop and demonstrate a procedure for simulating pyrolysis in complex geometries, and execute a coupled fire growth simulation using a 3D description of the condensed phase. Potential applications of this work include prediction of flame spread and heat release rate in complex geometries where multidimensional condensed-phase effects are significant, modeling of standardized fire tests ranging from small scale tests such as UL94 or ASTM D2863 to large-scale furnace tests, and better understanding flammability of anisotropic materials such as composites.

Additional work is necessary to determine under which conditions a one dimensional patchwise description of the condensed phase is adequate, and when a $3 \mathrm{D}$ description should be implemented. For instances where a 3D description of the condensed-phase is warranted, an important practical consideration not addressed by this work is determining an appropriate compromise between resolution and computational expense. Another area where additional work is needed is development of a computationally tractable approach to handle geometrical changes that occur as objects burn. As stated previously, one limitation of the Gpyro3D formulation presented here is that shrinkage due to reaction or density change is not considered. This means that surface regression does not occur, and the geometry/size of pyrolyzing or burning objects is invariant for the entire duration of the simulation. While this is not problematic for shortduration simulations, such as those involving ignition, it is not appropriate for long-duration simulations where considerable regression, volume change, or movement (melting, dripping, collapse) of the condensed-phase occurs.

\section{REFERENCES}

[1] Floyd, J., Forney, G., Hostikka, S., Korhonen, T., McDermott, R., and McGrattan, K., "Fire Dynamics Simulator Technical Reference Guide. Volume 1: Mathematical Model," NIST Special Publication 1018, December 2012

[2] http://code.google.com/p/firefoam-dev/

[3] Bal, N. and Rein, G., (2013) Relevant model complexity for non-charring polymer pyrolysis, Fire Safety Journal 61: 36-44, http://dx.doi.org/10.1016/j.firesaf.2013.08.015

[4] Bonnefoy, F., Gilot, P., and Prado, G., (1993) A three-dimensional model for the determination of kinetic data from the pyrolysis of beech wood, Journal of Analytical and Applied Pyrolysis 25: 387-394, http://dx.doi.org/10.1016/0165-2370(93)80057-7 
[5] Jia, F., Galea, E.R., and Patel, M.K., (1999) Numerical Simulation of the Mass Loss Process in Pyrolizing Char Materials, Fire and Materials 23: 71-78, http://dx.doi.org/10.1002/(SICI)10991018(199903/04)23:2<71::AID-FAM672>3.0.CO;2-5

[6] Yuen, R.K.K, Yeoh, G.H., de Vahl Davis, G., and Leonardi, E., (2007) Modelling the pyrolysis of wet wood - I. Three-dimensional formulation and analysis, International Journal of Heat and Mass Transfer 50: 4371-4386, http://dx.doi.org/10.1016/j.ijheatmasstransfer.2007.01.008

[7] Sudhakar, D.R. and Kolar, A.K., (2010) Transient Three-Dimensional Mathematical Model and Experimental Investigation of a Wet Devolatilizing Wood in a Hot Fluidized Bed, Energy \& Fuels 24: 4820-4832, http://dx.doi.org/10.1021/ef100394y

[8] McGurn, M.T., Ruggirello, K.P., and DesJardin, P.E., (2013) An Eulerian-Lagrangian Moving Immersed Interface Method for Simulating Burning Solids, Journal of Computational Physics 241: 364-387, http://dx.doi.org/10.1016/j.jcp.2013.01.045

[9] Lautenberger, C., "Generalized Pyrolysis Model for Combustible Solids," PhD Dissertation, Department of Mechanical Engineering, University of California at Berkeley, December 2007.

[10] Lautenberger, C. and Fernandez-Pello, A.C., (2009) Generalized Pyrolysis Model for Combustible Solids, Fire Safety Journal 44: 819-839, http://dx.doi.org/10.1016/j.firesaf.2009.03.011

[11] Lautenberger, C. \& Fernandez-Pello, A.C., (2009) A Model for the Oxidative Pyrolysis of Wood, Combustion and Flame 156: 1503-1513, http://dx.doi.org/10.1016/j.combustflame.2009.04.001

[12] Dodd, A.B., Lautenberger, C. \& Fernandez-Pello, A.C., (2009) Numerical Examination of TwoDimensional Smolder Structure in Polyurethane Foam, Proceedings of the Combustion Institute 32: 2497-2504, http://dx.doi.org/10.1016/j.proci.2008.06.196

[13] Patankar, S.V., Numerical Heat Transfer and Fluid Flow, Hemisphere Publishing Corporation, 1980.

[14] Reszka, P., "In-Depth Temperature Profiles in Pyrolyzing Wood," PhD Dissertation, University of Edinburgh, 2008.

[15] http://reaxengineering.com/trac/gpyro 